\title{
THE EFFECT OF ELECTROMAGNETIC STIMULATION OF THE POSTERIOR PARIETAL CORTEX ON EYE MOVEMENTS
}

\author{
P. T. G. ELKINGTON, G. K. KERR, J. S. STEIN \\ Oxford
}

\begin{abstract}
SUMMARY
The posterior parietal cortex probably plays a central role in the sensorimotor transformations needed to make an accurate saccadic eye movement to a visual target. In an attempt to disrupt the normal programming of saccades, we magnetically stimulated the posterior parietal cortex in human volunteers, $80 \mathrm{~ms}$ after a small target moved $5^{\circ}$ horizontally from the centre of a VDU screen. Saccadic eye movements were recorded and experimental trials were compared with control, unstimulated trials. Magnetic stimulation was triggered in $70 \%$ of the trials selected randomly. The main effects of stimulation were: increased divergence of the eyes before each saccade, greater latency of saccade onset, and a tendency to undershoot the target. These results support the hypothesis that the posterior parietal cortex is involved in the programming of accurate saccades to visual targets.
\end{abstract}

David Ferrier (1895) first proposed a visual role for the posterior parietal cortex (PPC). He found that stimulating the PPC in monkeys could evoke saccadic eye movements. This led him to make his famous mistake of concluding that the PPC was the primary visual cortex.' Nevertheless, a role for the PPC in eye movements was supported by the observations of Gordon Holmes (1918). He noted that many patients with lesions of the PPC were unable to make accurate eye movements.

With the advent of extracellular recording this hypothesis could be tested directly. The pioneering work of Mountcastle et al..$^{2}$ identified many neurons in Brodmann's area 7 that discharged before eye movements. Andersen et al. ${ }^{3}$ recorded throughout the PPC, and found a dense collection of saccade-related neurons in the lateral intraparietal area (LIP) of the inferior parietal lobule. Furthermore, Shubunati et al $^{4}{ }^{4}$ found that the area of the

From: University Laboratory of Physiology. Parks Road, Oxford OXI 3PT, UK.

Correspondence to: P. T. G. Elkington. Magdalen College, Oxford OXI GAU, UK
PPC with the lowest threshold for evoking saccades electrically was the LIP. However, the currents required were still fairly high in comparison with those needed to stimulate saccades from the frontal eye fields or the superior colliculus.

In this project we have used the recently reintroduced technique of magnetic stimulation of the brain to attempt to disrupt the accurate programming of saccades by the PPC. As early as 1896, d'Arsonval ${ }^{5}$ experimented with magnetic stimulation and reported the generation of magnetophosphenes. However, the technical difficulties involved in generating a sufficiently large localised magnetic field prevented much progress for the next 80 years. Recently, however, Barker et al. ${ }^{b}$ designed a magnetic stimulator that is able to stimulate the motor cortex effectively.

In magnetic stimulation a large capacitor discharges through a tightly wound coil producing a very rapid current change. This current change induces a strong magnetic field running at right angles to it. The pulse of magnetic field lasts for a few milliseconds.? In turn, the changing magnetic field induces an electric field (a voltage difference between two points), which then leads to eddy currents large enough to excite neural tissue.

So far, this technique has mainly been used to stimulate the motor cortex (e.g. Cohen et al.). ${ }^{8}$ Only a few studies have examined the association areas of the cortex. Two reports by Priori et al..$^{9.10}$ have suggested that transcranial magnetic stimulation of the PPC can delay saccadic reaction time.

\section{METHODS}

\section{Subjects}

The tests were performed on two healthy consenting male subjects, aged 30 and 35 years, with local ethical committee approval.

Setup

The subject sat $57 \mathrm{~cm}$ from a VDU screen. Head move- 
ments were prevented by a rigid bite bar and a support at the back of the neck. Eye movements were recorded with a Scalar 'Iris' infra-red eye movement recording system, mounted on a headset.

The magnetic stimulator used was a Magstim 200. It was set at $60 \%$ of maximum output. The stimulating coil used was the recently developed 'figure-of-eight' coil. This shape ensured that stimulation was restricted to a minimal area of cortex, and also made the possibility of unwanted contralateral stimulation very unlikely. The figure-of-eight design was first proposed by Ueno et al. ${ }^{\prime \prime}$ Two coils are wired side by side in such a way that the current passes in opposite directions in each coil. This means that the resultant current loops sum where the two coils approach each other, but not elsewhere (Fig. 1).

The point where the two coils met was positioned $3 \mathrm{~cm}$ posterior to the crown of the head and $3 \mathrm{~cm}$ laterally. The long axis of the figure of eight lay in the coronal plane. This meant that the induced magnetic field passed posteroanteriorly. Thus the induced current passed antero-posteriorly through the posterior parietal cortex.

\section{Protocol}

Each of the two subjects underwent two full trial sessions: one for left PPC stimulation, and one for right PPC stimulation. In each trial the subjects were asked to fixate a centrally displayed target. Two to 4 seconds after the experimenter warned them by saying 'ready', the target jumped $5^{\circ}$ horizontally to the left or right. The timing and direction of the jump were randomly selected. The target flashed there for $100 \mathrm{~ms}$. Magnetic stimulation of the PPC was triggered on $70 \%$ of the trials randomly selected, $80 \mathrm{~ms}$ after the target jump.

Trials were done in blocks of 20. Pre- and post-calibration runs were carried out at the start and end of each block. In the calibration runs the subject was asked to fixate the displayed target as it moved from the left-hand side of the screen to the right-hand side in five steps, and back again. This allowed the calibration factor (A/D units per degree) and the midline (zero) position to be calculated frequently.

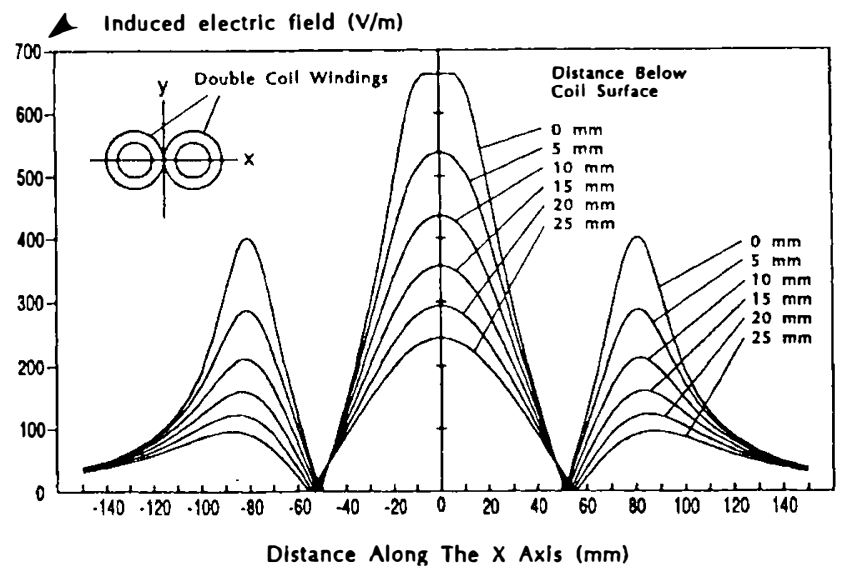

Fig. 1. Manufacturer's diagram showing peak induced electric field as a function of distance from the point of contact of the 'figure-of-eight' coil.
In each experiment, three blocks of trials were carried out for each hemisphere. Four variables were digitised at 1000 samples per second and then stored in an IBM computer. These were target position, stimulus trigger, and left and right eye positions. The eye movement recordings were then calibrated in terms of degrees of visual angle from the central fixation point. Any trial in which a blink occurred was eliminated at this point. This was easy as the blink artefacts showed very characteristically large rapid divergence followed by convergence.

For each eye the latency of saccade onset, peak saccade velocity, accuracy of the first saccade and final fixation point were computed. Also, the divergence of the eyes before the saccade onset and rate of divergence after the saccade were calculated. These variables were then analysed.

\section{Location of Stimulation}

The greatest problem with magnetic stimulation is that the magnetic field created is diffuse. Hence the precise area of the cortex that is being stimulated is uncertain. In the experiments reported here, there was scalp muscle contraction through direct stimulation by the magnetic field. However, there was only one instance of a subject reporting any other muscle contractions. This was in the thumb muscles of the thenar eminence, probably because the motor cortex was being stimulated. This occurred during one of the pilot runs, so the coil was then positioned further posteriorly. In no other trial was contraction evoked. Nor were any cutaneous, auditory or visual perceptual changes reported. This is good evidence that the coil did not stimulate visual, somaesthetic, auditory or motor cortices except in the one pilot trial. Thus the figureof-eight shaped coil seems to have ensured that the stimulation was limited to the intended area of the parietal cortex. Furthermore, the ovoid shape of the area of maximum excitation means that any unwanted contralateral stimulation was unlikely to have occurred.

\section{RESULTS}

An example of the effects of stimulation is shown in Fig. $2 \mathrm{a}-\mathrm{d}$. Five differences were noted during stimulated trials compared with controls: increased divergence of the eyes before the saccade was made, increased latency of saccade onset, decreased peak velocity of the saccade, hypometria of the saccade and rate of divergence of the eyes after fixation.

Only two of these changes were found to be statistically significant, however. These were divergence of the eyes before saccade onset and prolonged latency of saccade initiation. There was a tendency for the saccades to undershoot the target but this was not consistent enough to be statistically significant.

\section{Divergence of the Eyes Before the Saccade is Made}

There was a small divergence of the eyes before each sac- 
Control

a) Angle across the screen

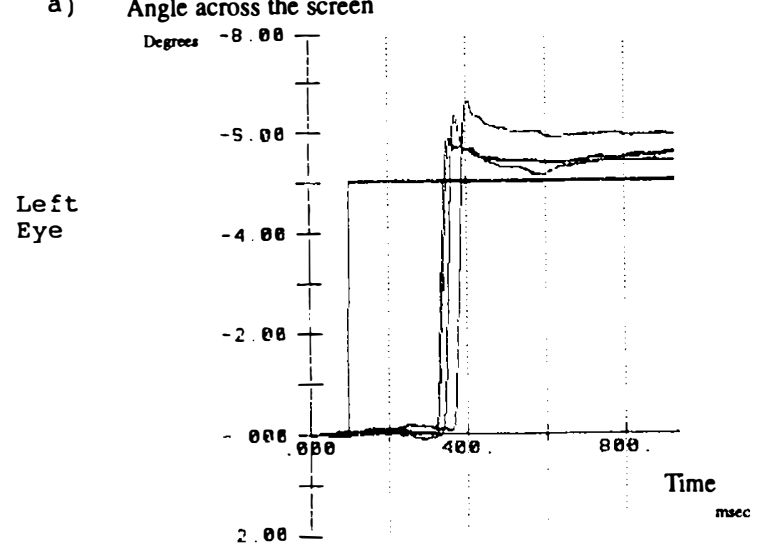

b) Angle across the screen

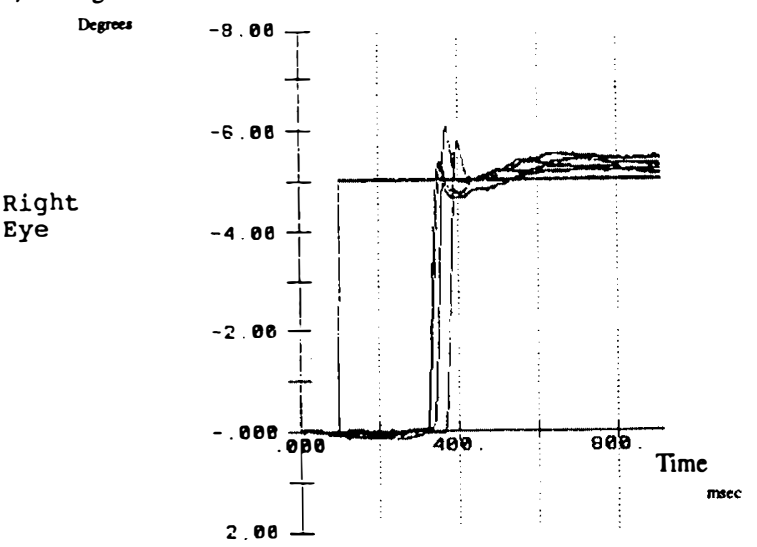

c)

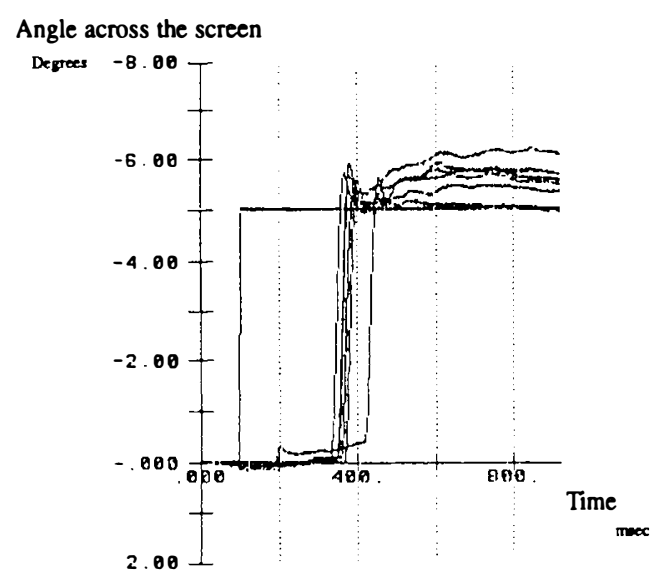

d) Angle across the screen

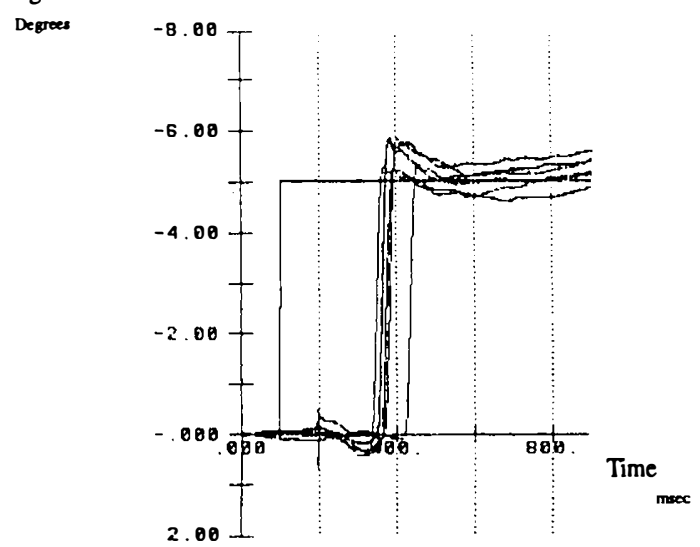

Fig. 2. Typical traces of eye movements during trials: (a) left eye, control: (b) right eye, control; (c) left eye, stimulation; (d) right eye, stimulation.

cade was made in the control trials, but this was significantly larger in the experimental trials. The sizes of the divergence differences are shown in Tables I and II. The differences were statistically significant in all but one trial $(p<0.05$; one-tailed $t$-test).

There was no clear correlation between the magnitude of the divergence and the direction of the saccade. However, the analysis was done in such a way that only the absolute difference in the position of fixation of the eyes was calculated. It is possible that one eye (e.g. that moving to the contralateral side) might have been diverging more, but this would not have been apparent in the results.

Table 1. Effects of right PPC stimulation on eye divergence before saccade onset (degrees)

\begin{tabular}{lcccc}
\hline & Control & Stimulation & Difference & $p$ value \\
\hline Subject l & & & & \\
Target left & 0.121 & 0.210 & 0.089 & 0.0246 \\
Target right & 0.129 & 0.216 & 0.087 & 0.0345 \\
Subject 2 & & & & \\
Target left & 0.341 & 0.819 & 0.478 & 0.0044 \\
Target right & 0.487 & 0.762 & 0.275 & 0.0285 \\
\hline
\end{tabular}

The table shows that right PPC stimulation significantly increased the eye divergence before saccade onset ( $p$, one-tailed $t$-test).

\section{Latency of Saccade Onset}

There was a marked prolongation of saccade latency in the stimulus trials. For subject 1 , the average increase was $30.3 \mathrm{~ms}$. There was also a progressive lengthening of the latencies of control trials during each experimental session as the subject gradually tired.

The latency of onset of saccades was found to have no significant difference between the left and right eyes. Therefore, only the average left eye latencies for each trial are included here. The latencies for each subject are shown in Tables III and IV.

There was one block in which the increased latency was

Table II. Effects of left PPC stimulation on eye divergence before saccade onset (degrees)

\begin{tabular}{lcccc}
\hline & Control & Stimulation & Difference & $p$ value \\
\hline Subject I & & & & \\
Target left & 0.125 & 0.236 & 0.111 & 0.0664 \\
Target right & 0.082 & 0.374 & 0.292 & 0.0089 \\
Subject 2 & & & & \\
Target left & 0.419 & 1.228 & 0.809 & 0.0007 \\
Target right & 0.298 & 1.024 & 0.726 & 0.0048 \\
\hline
\end{tabular}

The table shows that left PPC stimulation significantly increased eye divergence before saccade onset ( $p$, one-tailed $t$-test). 
Table III. Effects of right PPC stimulation on latency of saccade onset

\begin{tabular}{|c|c|c|c|c|}
\hline & Control & Stimulation & Difference & $p$ value \\
\hline \multicolumn{5}{|l|}{ Subject I } \\
\hline Target left & 213.5 & 243.6 & +30.1 & 0.0004 \\
\hline Target right & 207.4 & 234.9 & +27.5 & 0.0088 \\
\hline \multicolumn{5}{|l|}{ Subject 2} \\
\hline Target left & 299.0 & 237.1 & -61.9 & 0.0042 \\
\hline Target right & 300.9 & 304.3 & +3.4 & 0.4501 \\
\hline
\end{tabular}

The table shows that right PPC stimulation significantly increased latency of saccade onset in two trials ( $p$, one-tailed $t$-test).

Table IV. Effects of left PPC stimulation on latency of saccade onset

\begin{tabular}{lcccc}
\hline & Control & Stimulation & Difference & $p$ value \\
\hline Subject I & & & & \\
Target left & 211.7 & 234.2 & +22.5 & 0.0396 \\
Target right & 194.4 & 235.6 & +41.2 & 0.0002 \\
Subject 2 & & & & \\
Target left & 205.1 & 266.6 & +61.5 & 0.0082 \\
Target right & 208.8 & 320.0 & +111.2 & 0.0061 \\
\hline
\end{tabular}

The table shows that left PPC stimulation increased the saccade onset latency in all trials ( $p$. one-tailed $t$-test).

not seen. In this case, stimulating the right PPC of subject 2 , a decrease in the latency of onset was observed. The subject was very tired in this experiment and this was associated with a very much increased standard error.

Fig. 3 shows histograms of the distribution of saccade latencies for subject 1 . Clearly control trials fell into two distinct groups. In contrast, in the stimulus trials the shorter-latency group disappeared. Thus, stimulation probably increased the average latency not by slowing all saccades but by eliminating the fastest ones.

Analysis of the relation between the side of stimulation and the direction of target movement suggested that the difference in latency may have been more pronounced when the target moved to the contralateral side of the hemisphere being stimulated (Tables III and IV). For example, in the case of subject 1 , on stimulation of the left PPC the significance of the difference was $p=0.0002$ when the target moved to the right, but was only $p=$ 0.0396 when the target moved to the left.

\section{DISCUSSION}

The main findings of this experiment were that magnetic stimulation of the posterior parietal cortex before a saccadic eye movement led to significantly increased divergence of the eyes before saccade onset and increased latency of saccade initiation. The effect on latency was probably greater when the target of the saccade was contralateral to the stimulation.

Before these results are discussed, the effect that the magnetic stimulation has on the cortex should be considered. The stimulating coil was held so that the maximum induced current passed antero-posteriorly. The experiments of Rosler et al. ${ }^{12}$ have shown that the orientation of the coil is crucial when stimulating the motor cortex. They propose that the axons lying parallel to the induced current receive the greatest stimulation. In the
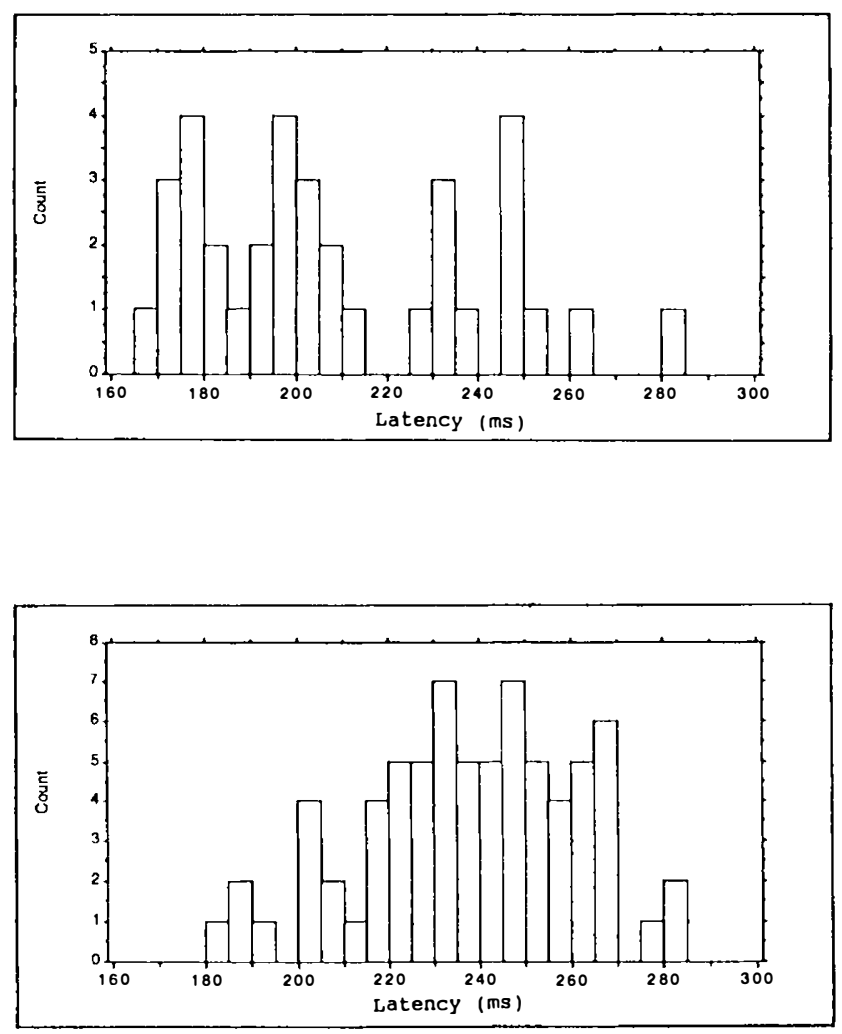

Fig. 3. Histogram showing the distribution of saccade latencies in subject 1 in control trials (upper) and stimulation trials (lower).

PPC many of the efferent axons run perpendicular to the surface of the cortex towards the basal ganglia and cerebellum. But one projection that is parallel to the surface is that to the frontal eye fields. Probably, therefore, this will have been maximally stimulated by the induced currents. Also the horizontal processes of interneurons within the cortex will have been stimulated. ${ }^{13}$ Thus, effects on the efferent projections and local effects on the PPC itself must be considered in interpreting the results.

Divergence of the eyes always occurs at the beginning of a saccade..$^{14}$ Its increase on stimulation suggests that accurate vergence control was disrupted. This finding helps to confirm the hypothesis that the PPC is important in the control of vergence eye movements. The clinical data of Fowler et al. ${ }^{15}$ showed that vergence control for small targets was particularly impaired in patients with lesions of the PPC. This suggests that the PPC controls the fine tuning of the vergence system, whereas the gross control of these movements may be effected by other areas. As the PPC receives afferents from extrastriate areas that will signal the distance of visual targets, it is ideally placed to use this information to control target fixation in depth accurately.

The delay of saccadic onset clearly indicates that stimulation affected some aspect of the processing of the visual information required to program saccades. The bimodal distribution of latencies of control, unstimulated saccades (Fig. 3) suggests that there are two characteristic latencies for the programming of saccades. For subject 1 these were centred around $190 \mathrm{~ms}$ and $240 \mathrm{~ms}$. In the stimulation 
trials there was only a single grouping, with a mean of $237 \mathrm{~ms}$. It would seem, therefore, that stimulation eliminated the faster rate of saccade programming. This would explain the decrease in variability of saccade latencies during stimulation.

This effect on saccade latency may result either from stimulating PPC efferents to the frontal eye fields or direct inhibitory efferents on the saccade generator in the brainstem, ${ }^{16}$ or it could be because of a direct action on the PPC itself. We propose that the increase in the average latency of saccades is due to a direct effect on the PPC. An inhibition of the brainstem saccade generator is unlikely as efferents projecting there from the PPC run perpendicular to the surface of the cortex. Thus they are unlikely to be stimulated much by the currents induced by the magnetic field. Frontal eye field involvement is unlikely as Fox et al. ${ }^{17}$ showed in studies using positron emission tomography that short-latency saccades in response to a visual target (as opposed to spontaneous saccades), such as the ones made in this experiment, may be generated without the involvement of the frontal eye fields.

We delivered magnetic stimulation around the time that PPC neurons would be expected to respond to the flashed visual target. ${ }^{3}$ Therefore, PPC cells were probably refractory as a result of their recent depolarisation by the stimulation when the visual information needed to generate the saccade arrived from the extrastriate visual areas, thus preventing rapid programming of the next saccade. This would explain why the short latencies seen in the control trials were eliminated during stimulation. Probably the longer latency saccades were not affected by the stimulation because the cells were no longer refractory. Thus only the short-latency saccades were interrupted and after stimulation all saccades came to have the same latency as the slower group of control saccades.

As expected, the increase in latency was greatest when the target moved to the contralateral side. This was probably because each PPC receives most of its visual information from the contralateral hemifield. It was more interesting that there was also a marked delay of saccades to the side ipsilateral to the stimulation. This might result from the induced current spreading to the contralateral hemisphere so that it partially stimulated the opposite PPC. However, this was unlikely as the figure-of-eight coil was specifically designed to stimulate only locally.

Lesions of the PPC result primarily in contralateral visuospatial defects, but there are also some bilateral effects which are probably the result of communication between the two PPC via the corpus callosum. Therefore, the slowing of saccades to the ipsilateral side may result from the stimulated hemisphere influencing its pair via the corpus callosum. Although each PPC primarily represents the contralateral field, there is a considerable amount of overlap between the two.

The results of this study support the conclusion that the
PPC plays a central role in the programming of saccadic eye movements. Each PPC is primarily involved in controlling saccades to the contralateral side but there is considerable overlap between the two cortices. Both hemispheres seem to be concerned in the control of accurate vergence eye movements.

Key words: Magnetic stimulation, Posterior parietal cortex, Saccades.

\section{REFERENCES}

1. Glickstein MH: Ferrier's mistake. TINS 1985, 8: 341-4.

2. Mountcastle VB, Lynch JC, Georgopoulos A, Sakata H, Acuna C: Posterior parietal association cortex of the monkey: command function for operations in extrapersonal space. J Neurophysiol 1975, 38: 871-907.

3. Andersen RA, Essick GK, Siegel RM: The encoding of spatial location by posterior parietal neurons. Science 1985, 230: $456-8$.

4. Shubutani H, Sakata H, Hyvarinen J: Saccade and blinking evoked by microstimulation of the posterior parietal association area of the monkey. Exp Brain Res 1984, 55: 1-8.

5. d'Arsonval A. Dispositifs pour la mesure des courants alternatifs de toutes fréquences. $C R$ Soc Biol 1896, 2: 450-1.

6. Barker AT, Jalinous R, Freeston IL: Non-invasive stimulation of the human motor cortex. Lancet 1985, ii: 1106-7.

7. Jalinous R: Technical and practical aspects of magnetic nerve stimulation. J Clin Neurophysiol 1991, 8: 10-25.

8. Cohen LG, Hallet M, Lelli S: Non-invasive mapping of human motor cortex with transcranial magnetic stimulation. In: Chrokroverty S, ed. Magnetic stimulation in clinical neurophysiology. London, Butterworth, 1990: 113-21.

9. Priori A, Bertolasi L, Rothwell JC, Day BL, Marsden CD: Human saccadic reaction time is delayed by transcranial magnetic stimulation. J Physiol 1990, 435: 54P.

10. Priori A, Bertolasi L, Rothwell JC, Day BL, Marsden CD: Evidence that transcranial magnetic stimulation delays saccadic eye movements by interfering with activity in oculomotor areas of the cortex. $J$ Physiol 1991, 438: 15P.

11. Ueno S, Tashiro T, Harada K: Localised stimulation of neural tissue in the brain by means of a paired configuration of time-varying magnetic fields. J Appl Physiol 1988, 64: $5862-4$.

12. Rosler KM, Hess CW, Heckmann R, Ludin HP: Significance of the shape and size of the stimulating coil in magnetic stimulation of the human motor cortex. Neurosci Lett 1989, 100: 347-52.

13. Amassian VE, Stewart M, Quirk GJ, Rosenthal JL: Physiological basis of motor effects of a transient stimulus to the cerebral cortex. Neurosurgery 1987, 20: 148-55.

14. Erkelens CJ, Steinman RM, Collewijm H: Ocular vergence under natural conditions. II. Gaze shifts between real targets differing in distance and direction. Proc $R$ Soc Lond [Biol] 1989, 236: 441-65.

15. Fowler S, Munro N, Richardson A, Stein J: Vergence control in patients with lesions of the posterior parietal cortex. J Physiol 1989, 417: 92P.

16. Komatsu H, Wurtz RH: Modulation of pursuit eye movements by stimulation of cortical areas MT and MST. J Neurophysiol 1989, 62: 31-47.

17. Fox PT, Fox JM, Raichle ME, Burde RM: The role of the cerebral cortex in the generation of voluntary saccades: a positron emission tomographic study.J Neurophysiol 1985, 54: 348-69. 